\title{
Macro- and microvascular alterations in patients with metabolic syndrome: sugar makes the difference
}

\author{
Panagiota Pietri, Charalambos Vlachopoulos, Gregory Vyssoulis, Nikolaos Ioakeimidis and \\ Christodoulos Stefanadis
}

\begin{abstract}
Metabolic syndrome (MS) is associated with adverse cardiovascular events, although its prognostic significance over and beyond the clustering risk factors is controversial. Moreover, there are no data on the possible differentiation of target organ damage among patients with MS according to the grade of its distinct components. We studied 500 hypertensive patients with MS and we assessed vascular damage according to glucose metabolic status (1, normal glucose metabolism (NG); 2 , impaired fasting glucose (IFG); 3, impaired glucose tolerance (IGT); and 4, diabetes mellitus II (DM II)). Macrovascular damage was assessed with arterial stiffness by measuring carotid-femoral pulse wave velocity (PWV). Microvascular damage was assessed with albumin excretion by estimating the albumin-creatinine ratio (ACR). There was a significant progressive increase in PWV from group 1 to group 4 (from 7.97 to 8.83 to 8.94 to $10.27 \mathrm{~m} \mathrm{~s}^{-1}$, respectively) that remained statistically significant even after adjustment for several confounders $(\boldsymbol{P}<0.001)$. Similar trends were also observed for ACR (from 27.44 to 29.94 to 36.26 to $\left.73.07 \mathrm{mgg}^{-1}, P<0.001\right)$. In multiple regression analysis, both PWV and ACR were independently related to glucose metabolic status $(P=0.001$ and $P<0.001$, respectively). Vascular alterations among patients with MS differ according to the grade of glucose dysregulation. Considering the adverse prognostic role of arterial stiffness and microalbuminuria, it might be argued that the cardiovascular risk is not homogeneously distributed among patients with MS but is largely determined by glucose metabolic status.
\end{abstract}

Hypertension Research (2014) 37, 452-456; doi:10.1038/hr.2013.148; published online 31 October 2013

Keywords: arterial stiffness; cardiovascular risk; glucose metabolism; metabolic syndrome; microalbuminuria

\section{INTRODUCTION}

Metabolic syndrome (MS) is associated with increased cardiovascular risk, ${ }^{1-4}$ although its incremental prognostic value, over and beyond its clustering risk factors, has been questioned. Indeed, previous data have shown that distinct components of MS, such as impaired fasting glucose (IFG), impaired glucose tolerance (IGT), low high-density lipoprotein cholesterol and microalbuminuria, predict cardiovascular mortality with equal or higher hazard ratios than MS itself as a unique entity. ${ }^{5}$ Moreover, it has been suggested that specific combinations of the components of MS may be more powerful predictors of mortality as compared with others. ${ }^{6}$ Similar discrepancies also exist for the underlying pathophysiological mechanisms linking MS with cardiovascular mortality. ${ }^{7-10}$

Arterial stiffness is an independent predictor of cardiovascular events in several populations, ${ }^{11}$ including patients with glucose intolerance. ${ }^{12} \mathrm{We}^{13}$ and others ${ }^{14-19}$ have shown that MS is associated with increased arterial stiffness, regardless of the applied definition criteria. Arterial stiffness may explain the increased cardiovascular risk associated with MS, but whether arterial stiffness may differ among patients with MS, according to the level of its distinct components, and specifically the glucose status, has not been fully explored.
Microalbuminuria is both a marker of endothelial dysfunction ${ }^{20}$ and a predictor of cardiovascular events. ${ }^{21}$ Microalbuminuria has been closely related to MS and was among its constituents according to the first definition criteria established by World Health Organization (WHO). ${ }^{22}$ Whether the level of microalbuminuria (and thus, the potential cardiovascular risk) may differ among patients with MS is not well clarified.

The aim of the present study was to investigate whether there is a difference in macro- and microvascular organ damage in patients with MS according to glucose metabolic status. For this purpose, we studied 500 patients with metabolic syndrome, whom we divided into four groups according to glucose status, and we assessed arterial stiffness and microalbuminuria as markers of large artery and endothelial dysfunction, respectively.

\section{METHODS}

Study population

We studied 500 consecutive never-treated hypertensive patients with metabolic syndrome. Office blood pressure (BP) was measured by a mercury sphygmomanometer, taking at least three measurements spaced by $1 \mathrm{~min}$, allowing the patients to rest for $10 \mathrm{~min}$ before examination. According to the guidelines of 
the European Society of Hypertension, measurements of systolic BP (SBP) $\geqslant 140 \mathrm{~mm} \mathrm{Hg}$ and/or diastolic BP $\geqslant 90 \mathrm{~mm} \mathrm{Hg}$ were considered as systolic and diastolic arterial hypertension, respectively. Pulse pressure (PP) was calculated as SBP minus diastolic BP and mean BP (mean arterial pressure (MAP)) as diastolic BP plus $1 / 3 \mathrm{PP}$.

The determination of MS was based on Adult Treatment Panel III (ATP III) criteria: three or more of the following, including hypertension: 1, waist circumference $>102 \mathrm{~cm}$ for men and $>88 \mathrm{~cm}$ for women; 2, serum triglycerides $\geqslant 150 \mathrm{mg} \mathrm{dl}^{-1} ; 3$, high-density lipoprotein cholesterol $<40 \mathrm{mg} \mathrm{dl}^{-1}$ for men or $<50 \mathrm{mg} \mathrm{dl}^{-1}$ for women; and 4, fasting glucose level $\geqslant 100 \mathrm{mg} \mathrm{dl}^{-1}$. Patients with glucose levels $>100 \mathrm{mg} \mathrm{dl}^{-1}$ were subject to an oral glucose tolerance test with $75 \mathrm{~g}$ of glucose. According to the oral glucose tolerance test results, patients with MS were divided into four groups: 1, normal glucose metabolism (NG; fasting glucose levels $<100 \mathrm{mg} \mathrm{dl}^{-1}$ ); 2 , IFG (fasting glucose levels between 100 and $125 \mathrm{mg} \mathrm{dl}^{-1}$ and $2 \mathrm{~h}$ postload glucose $\left.<140 \mathrm{mg} \mathrm{dl}^{-1}\right)$; 3 , IGT $(2 \mathrm{~h}$ postload glucose between 140 and $199 \mathrm{mg} \mathrm{dl}^{-1}$ ); and 4, diabetes mellitus II (DM II; fasting glucose levels $\geqslant 126 \mathrm{mg} \mathrm{dl}^{-1}$ on two measurements or $2 \mathrm{~h}$ postload glucose $\geqslant 200 \mathrm{mg} \mathrm{dl}^{-1}$ or use of antidiabetic drugs). Diabetic patients on insulin therapy were excluded from the study. Antidiabetic drugs (mainly metformin-based regimens) were used by $16 \%$ of diabetic patients.

Body weight and height were measured in all subjects and body mass index (BMI) was calculated. Smoking status was assessed by recording the habit of smoking. Patients with overt cardiovascular disease, severe valvular disease, cerebrovascular disease, peripheral arterial disease, systemic disorders, acute infections and moderate or severe chronic kidney disease (estimated glomerular filtration rate $\leqslant 50 \mathrm{ml} \mathrm{min}^{-1}$ per $1.73 \mathrm{~m}^{2}$ ) were excluded from the study. Use of statins, aspirin or other anti-inflammatory agents was also an exclusion criterion.

All patients gave their informed consent to participate in the study that was approved by the Local Ethics Committee.

\section{Evaluation of arterial stiffness}

Arterial stiffness was evaluated noninvasively by the measurement of carotidfemoral pulse wave velocity (PWV). Carotid-femoral PWV, an established marker of aortic stiffness, ${ }^{11,23}$ was obtained using a validated noninvasive device (Complior, Artech Medical, Pantin, France) ${ }^{24}$ that allows online pulse wave recording and automatic calculation of PWV. Two different pressure waveforms were obtained simultaneously at two sites: the right carotid and femoral artery. The time delay $(t)$ between the feet of the recorded proximal and distal waves was automatically calculated. For the carotid-femoral segment, the distance $(d)$ was estimated by subtracting the distance between carotid location and sternal notch from the distance between sternal notch and femoral site. The mean PWV of at least 10 consecutive pressure waveforms was calculated for further analysis.

All measurements were conducted in the morning, with the individual in the supine position, in a quiet environment. Participants were requested to abstain from tobacco, coffee and food at least $5 \mathrm{~h}$ and from alcohol $12 \mathrm{~h}$ before the examination.

\section{Laboratory assays}

Blood samples were taken between 0800 and $0900 \mathrm{~h}$ in the morning after an overnight fast. Routine biochemical parameters were determined with standard techniques by an autoanalyzer. Kidney function was assessed by estimated glomerular filtration rate using the MDRD formula. Urine albumin concentration was measured after a 24 -h urine collection by immunonephelometry (Dade Behring, Marburg, Germany) and albumin-to-creatinine ratio (ACR) was calculated.

Insulin levels were measured with a microparticle enzyme immunoassay method (Abbott, Tokyo, Japan) and insulin resistance was estimated by applying the homeostasis model assessment (HOMA). Inflammatory status was assessed by measurement of high-sensitivity C-reactive protein (hsCRP) using an immunonephelometric method (Dade Behring High Sensitivity CRP assay; Dade Behring). The upper normal limit of hsCRP was $3 \mathrm{mgl}^{-1}$ with intra- and interassay coefficients of variation of $<4.4 \%$ and $5.7 \%$, respectively). Homocysteine was used as a prothrombotic marker and its levels were measured by enzyme immunoassay method (enzyme-linked immunosorbent assay, Abbott).

\section{Statistical analysis}

All continuous variables are presented as mean \pm s.d., whereas categorical variables are presented as percentages. The hsCRP, homocysteine and ACR were log-transformed because of their skewed distribution.

Difference in baseline characteristics among the four groups of patients was studied using analysis of variance or $\chi^{2}$ for the categorical variables, respectively. Analysis of covariance was used to detect differences in PWV and ACR values according to glucose metabolic status after adjustment for age, gender, smoking, BMI, total cholesterol, MAP (or SBP), heart rate and estimated glomerular filtration rate (used in the model of ACR only).

Linear regression analysis was applied in the whole cohort to determine the independent relationship of PWV and ACR with glucose status (as continuous variable) after adjustment for age, gender, smoking, BMI, total cholesterol, MAP, heart rate, HOMA, loghsCRP and loghomocysteine.

Exact $P$-values of $<0.05$ were considered as statistically significant. Data analysis was performed with SPSS software, version 17.0 (Chicago, IL, USA).

\section{RESULTS}

The baseline characteristics of the four groups of patients are presented in Table 1. The patients with deteriorating glucose metabolism were older than patients with NG values. IGT and DM II patients were more obese than patients with NG and IFG. The mean blood pressure did not differ between groups, although SBP was higher in patients with DM II than the other groups, and in patients with IGT than in those with NG. Regarding lipid status, total cholesterol was higher in patients with IGT than the other groups, whereas interestingly a gradual increase in high-density lipoprotein values was observed from patients with NG to IFG, IGT and DM II. Deterioration of glucose metabolism was accompanied by a gradual increase in insulin resistance (as estimated by HOMA) and hsCRP levels. In contrast, homocysteine levels were higher only in the DM II group. Finally, kidney function showed a progressive decline as glucose metabolism deteriorated.

PWV showed a significant, gradual increase from group 1 to group 4 (from $7.97 \pm 1.37$ to $8.83 \pm 1.68$ to $8.94 \pm 1.26$ to $10.27 \pm 2.09 \mathrm{~m} \mathrm{~s}^{-1}$, respectively) (Figure 1), whereas the relationship of PWV with glucose status remained significant even after adjustment for several confounders $(P<0.001)$. In the post hoc analysis, there was a significant difference in all comparisons between groups $(P<0.001)$ apart from the difference between patients with IFG and IGT $(P=$ nonsignificant $)$. In linear regression analysis, PWV was independently related to glucose metabolic status (as continuous variable) after adjustment for age, gender, BMI, MAP, heart rate, total cholesterol, HOMA, loghsCRP and loghomocysteine (Table 2). Insertion of SBP (instead of MAP) to the model did not alter the significant independent association of PWV with glucose status.

Similar trends were also observed for ACR values across the four groups of patients (ACR: from $27.44 \pm 31.92$ to $29.94 \pm 26.35$ to $36.26 \pm 34.34$ to $73.07 \pm 70.15 \mathrm{mgg}^{-1}$, respectively; Figure 1) that remained statistically significant even after adjustment for the confounders $(P<0.001)$. In the post hoc analysis, all between-group comparisons were significant $(P=0.04$ for NG-IFG, $P<0.01$ for the other comparisons), apart from the difference in ACR between IFG and IGT that was nonsignificant ( $P=$ nonsignificant). An independent association between ACR and glucose metabolic status was demonstrated in linear regression analysis after adjustment for the above-mentioned confounders (Table 2).

Finally, PWV and ACR were independently related after adjustment for age, gender, BMI, MAP, total cholesterol, HOMA, glucose status, 
Table 1 Baseline characteristics and their differences among the four groups of patients with metabolic syndrome

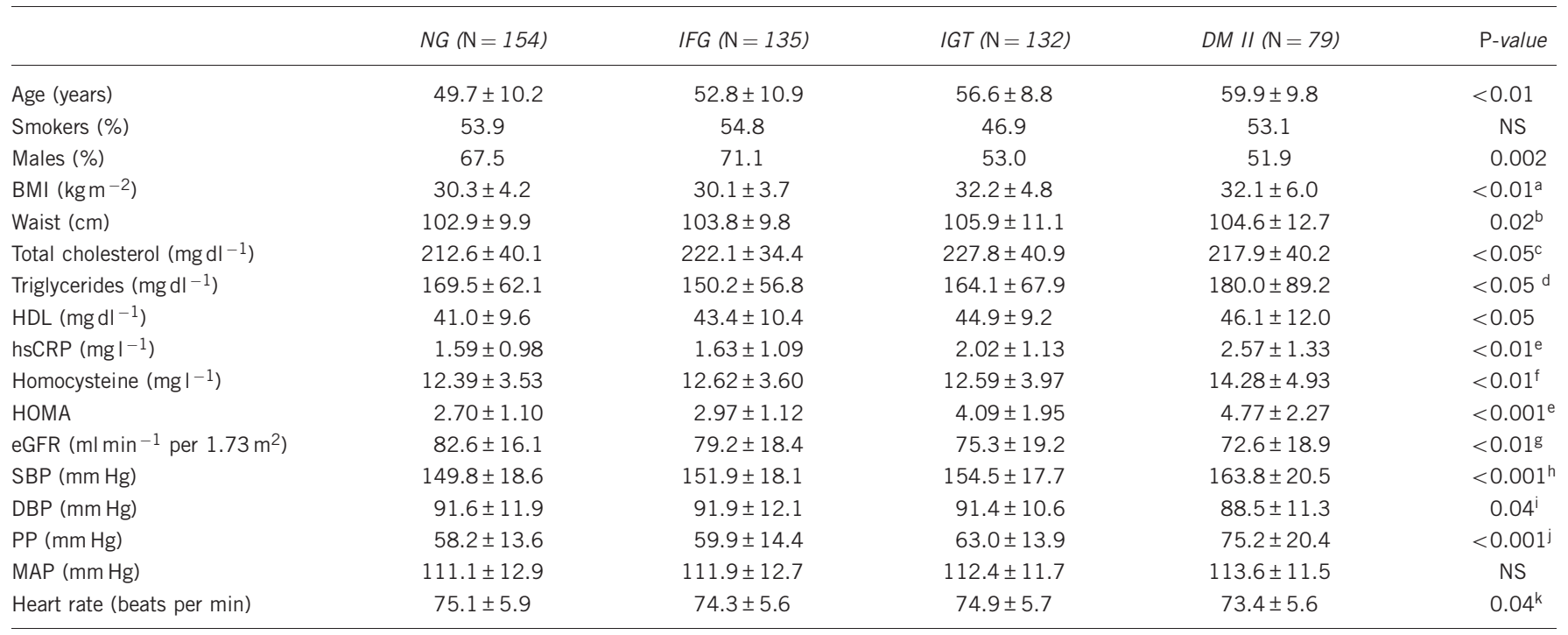

Abbreviations: BMI, body mass index; DBP, diastolic blood pressure; DM II, diabetes mellitus II; eGFR, estimated glomerular filtration rate; HDL, high-density lipoprotein; hsCRP, high sensitivity C-reactive protein; HOMA, homeostasis model assessment; IFG, impaired fasting glucose; IGT, impaired glucose tolerance; MAP, mean arterial pressure; NG, normal glucose metabolism;

NS, nonsignificant; PP, pulse pressure; SBP, systolic blood pressure.

aDifference between NG-IGT, NG-DM II and IFG-IGT.

bDifference between NG and IGT.

'Difference between NG-IFG and NG-IGT.

${ }^{d}$ Difference between NG-IFG and IFG-DM II.

eDifference between all group comparisons except for NG-IFG.

${ }^{f}$ Difference between NG-DM II and IFG-DM II.

gDifference between NG-IGT, NG-DM II and IFG-DM II.

hDifference between DM II and each of the other three groups (also the difference between IGT-NG was significant at the level of $P=0.03$ ).

iDifference between NG and DM II.

iDifference between DM II and each of the other three groups (also the difference between IGT-NG was significant at the level of $P=0.007$ ).

kDifference between NG and DM II.
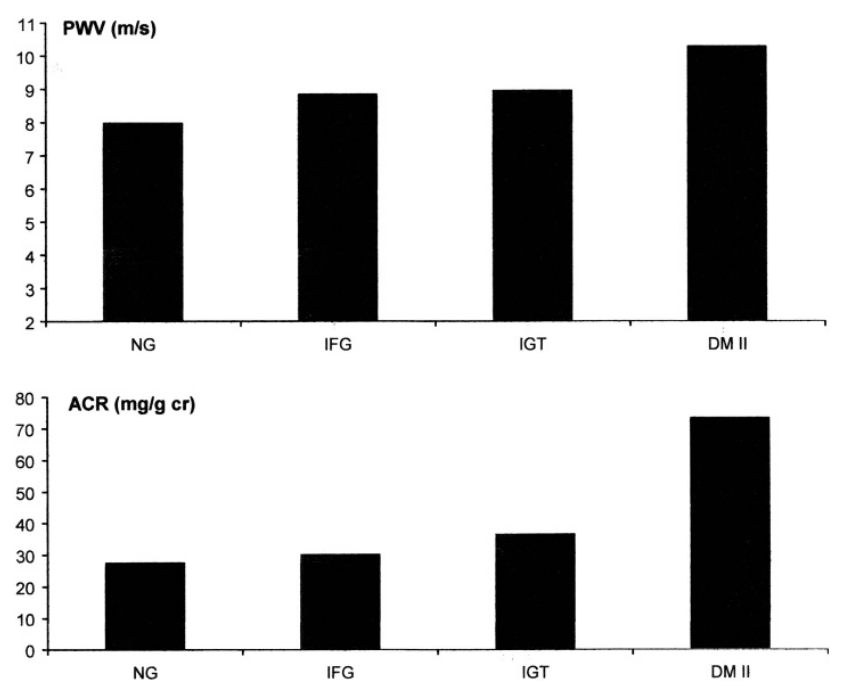

Figure 1 Progressive increase in pulse wave velocity (PWV, upper panel) and albumin-to-creatinine ratio (ACR, lower panel) according to the gradually abnormal glucose metabolism in 500 patients with metabolic syndrome. DM II, diabetes mellitus II; IFG, impaired fasting glucose; IGT, impaired glucose tolerance; NG, normal glucose metabolism.

loghsCRP and loghomocysteine $(P<0.001)$. In contrast, no significant independent association was established between PP (the pulsatile component of BP and an indirect marker of arterial stiffness) and ACR after adjustment for the same confounders $(P=0.06)$.
Table 2 Independent associations of PWV and ACR with glucose metabolic status (as continuous variable) in the whole population after adjustment for age, gender, smoking, BMI, total cholesterol, hsCRP, homocysteine, HOMA, MAP and heart rate

\begin{tabular}{lcc}
\hline & $\beta$-Coefficient & P-value \\
\hline PWV & & \\
Age & 0.27 & $<0.001$ \\
MAP & 0.18 & $<0.001$ \\
logACR & 0.16 & $<0.001$ \\
Glucose status & 0.14 & 0.001 \\
loghsCRP & 0.14 & 0.001 \\
HOMA & 0.10 & 0.01 \\
loghomocysteine & 0.08 & 0.03 \\
$R^{2}=0.40$ & & \\
LogACR & & $<0.001$ \\
loghsCRP & 0.34 & $<0.001$ \\
Glucose status & 0.19 & $<0.001$ \\
MAP & 0.16 & $<0.001$ \\
loghomocysteine & 0.16 & \\
$R^{2}=0.30$ & &
\end{tabular}

Abbreviations: ACR, albumin-to-creatinine ratio; BMI, body mass index; HOMA, homeostasis model assessment; hsCRP, high-sensitivity C-reactive protein; MAP, mean arterial pressure; PWV, carotid-femoral pulse wave velocity.

\section{DISCUSSION}

The present study demonstrates a significant differentiation in arterial stiffness and microalbuminuria among patients with MS according to their glucose metabolic status. Given that arterial stiffness and 
microalbuminuria reflect the macro- and microvascular organ damage and considering their strong predictive value for future cardiovascular events, the present findings suggest that the cardiovascular risk related to MS is not homogeneously distributed among all patients with MS; instead, it is largely determined by the grading effect of its components and, specifically, by glucose metabolic status.

\section{Clinical implications}

There is a constant debate on the independent prognostic role of MS over and beyond its clustering risk factors. Moreover, the increased cardiovascular risk associated with it differs according to the type of component combinations. ${ }^{6}$ We add further evidence on this issue. Indeed, the fasting glucose level of $>100 \mathrm{mg} \mathrm{dl}^{-1}$ as a component of MS implies that patients with NG as well as patients with IFG, IGT and DM II may all be included in the syndrome. However, our study shows that vascular damages and their corresponding risk (given the prognostic role of aortic PWV and microalbuminuria) differ among patients with MS according to the grade of abnormal glucose metabolism, and thus patients can be reclassified into a different category of cardiovascular risk within MS. In line with our observations are the results of a previous study showing that large artery thickness and stiffness (indices of vascular damage) differ in patients with MS according to distinct combinations. ${ }^{25}$ Accordingly, glucose metabolism may be used as a simple and cost-effective tool for the reclassification of patients according to this individualized risk, further aiding implementation of preventive strategies.

Macro- and microvascular damage as possible underlying mechanisms for the association of MS with cardiovascular risk PWV showed a progressive increase from patients with NG to patients with IFG, IGT and DM II. Systolic blood pressure is a powerful determinant of arterial stiffness, and it could be argued that the difference in its levels between some of the groups of our population might mediate part of the association of PWV with glucose status. Moreover, novel markers of inflammation, insulin resistance and prothrombotic state (namely, homocysteine) have all been independently associated with increased arterial stiffness. ${ }^{18,26-28}$ Considering that their levels showed a different distribution among the studied groups, it might be suggested that these factors may be responsible for the association of PWV with the glucose status. However, in multiple regression analysis the relationship of PWV with glucose metabolic status was independent of classic risk factors such as age, blood pressure (both SBP and MAP), total cholesterol, smoking and obesity, as well as novel risk factors such as hsCRP, HOMA and homocysteine.

Microalbuminuria has been identified as a marker of endothelial dysfunction..$^{20}$ Moreover, albumin excretion, even at levels well below the threshold for microalbuminuria definition, has been associated with increased cardiovascular risk in both diabetic and nondiabetic individuals. ${ }^{21}$ Microalbuminuria may mediate part of the increased cardiovascular risk associated with MS, and at a stage it was encompassed as a component of the syndrome according to the definition by WHO. Apart from the traditional risk factors such as hypertension and hyperglycemia, microalbuminuria is related to lowgrade inflammation and homocysteine ${ }^{29-33}$ that might have served as potent mediators to the association of albumin excretion with glucose metabolic status that we demonstrated in our study. Although this association remained significant after adjustment for inflammatory and prothrombotic markers, thus illustrating the ability of glucose metabolism per se to determine albumin excretion, contribution of such factors is likely. In any case, our study is a cross-sectional one and no pathophysiological or cause-and-effect relationships can be established.

The link between macro- and microvascular organ damage in MS In the present study, albumin excretion and PWV were independently associated, whereas the trends in their changes according to the glucose metabolic status were similar, both underlying the parallel adaptation of large arteries and endothelium (functioning as a vascular continuum) to the abnormal glucose metabolism. Arterial stiffening leads to increased pulsatile stress on the small arteries of the kidney that further leads to endothelial damage and to albumin excretion from the glomerulus. ${ }^{34,35}$ Studies have shown that $24 \mathrm{~h}$ PP is a better predictor of microalbuminuria than $24 \mathrm{~h}$ arterial stiffness index. $^{36}$ However, in our study, whereas PWV and ACR were independently related, no significant association was demonstrated between PP and ACR. This lack of association may be related either to the fact that PP measured by sphygmomanometry is a crude measure of arterial pulsatility or the possibility that the central (that is, the aortic) $\mathrm{PP}^{34}$ rather than the peripheral $\mathrm{PP}$, that we measured in our study is a more powerful determinant of renal damage. It should be noted, however, that other mechanisms, apart from the increased pulsatile stress, could explain the link between large artery stiffness and small vessel damage in the kidney. Afferent arterioles protect the glomerulus, through their regulated vasoconstriction, from damage induced by increases in systemic pressure. Defective arterial autoregulation (and thus increased hydraulic load imposed on the nephrons and resultant damage) may be related to increased arterial stiffness. ${ }^{37}$ Finally, inflammation, which is etiologically related to both endothelial dysfunction and large artery stiffening, may also provide an explanatory basis. ${ }^{38,39}$ Nevertheless, future studies are warranted to elucidate the underlying mechanisms of the association between aortic stiffness and small vessel injury.

We acknowledge some limitations of our study. Our population comprised patients with MS who were all treatment-naive hypertensive subjects. Whether these findings also apply to normotensive patients with MS can only be speculated.

\section{CONCLUSIONS}

The present study highlights, for the first time, the pivotal role of glucose metabolism per se in the differentiation of arterial stiffness and albumin excretion among patients with MS. Accordingly, the cardiovascular risk conveyed by MS may not be equally distributed among patients, and thus the management of patients with MS should be individualized, guided by the assessment of the burden of each component and, particularly, glucose metabolic status.

\section{CONFLICT OF INTEREST}

The authors declare no conflict of interest.

1 Dekker J, Girman C, Rhodes T, Nijpels G, Stehouwer C, Bouter L, Heine R. Metabolic syndrome and 10-year cardiovascular disease risk in the Hoorn Study. Circulation 2005; 112: 666-673.

2 Lakka H-M, Laaksonen D, Lakka T, Niskanen L, Kumpusalo E, Toumilehto J, Salonen J. The metabolic syndrome and total and cardiovascular disease mortality in middle-aged men. JAMA 2002; 288: 2709-2726.

3 McNeill AM, Rosamond W, Girman S, Golden SH, Schmidt M, East H, Ballantyne C, Heiss G. The metabolic syndrome and 11-year risk of incident cardiovascular disease in the Atherosclerosis Risk in Communities Study. Diabetes Care 2005; 28: 385-390.

4 Scuteri A, Najjar S, Morrell C, Lakatta E. The metabolic syndrome in older individuals: prevalence and prediction of cardiovascular events. The Cardiovascular Health Study. Diabetes Care 2005; 28: 882-887. 
5 Wang J, Ruotsalainen S, Moilanen L, Lepistö P, Laakso M, Kuusisto J. The metabolic syndrome predicts cardiovascular mortality: a 13-year follow-up study in elderly nondiabetic Finns. Eur Heart J 2007; 28: 857-864.

6 Guize L, Thomas F, Pannier B, Bean K, Jego B, Benetos A. All-cause mortality associated with specific combinations of the metabolic syndrome according to recent definitions. Diabetes Care 2007; 30: 2381-2387.

7 Ridker P, Buring J, Cook N, Rifai N. C-reactive protein, the metabolic syndrome and risk of incident cardiovascular events. Circulation 2003; 107: 391-397.

8 Sattar N, Gaw A, Scherbakova O, Ford I, O'Reilly D, Haffner S, Isles C, Macfarlane P, Packard C, Cobbe S, Shepherd J. Metabolic syndrome with and without C-reactive protein as a predictor of coronary heart disease and diabetes in the West of Scotland Coronary Prevention Study. Circulation 2003; 108: 414-419.

9 Langenberg C, Bergstrom J, Scheidt-Nave C, Pfeilschifter J, Barrett-Connor E. Cardiovascular death and the metabolic syndrome. Role of adiposity-signaling hormones and inflammatory markers. Diabetes Care 2006; 29: 1363-1369.

10 Anand S, Yi Q, Gerstein H, Lonn E, Jacobs R, Vuksan V, Teo K, Davis B, Montague P, Yusuf S. For the Study of Health Assessment and Risk in Ethnic groups (SHARE) and Study of Health Assessment and Risk Evaluation in Aboriginal Peoples (SHARE-AP) Investigators. Relationship of metabolic syndrome and fibrinolytic dysfunction to cardiovascular disease. Circulation 2003; 108: 420-425.

11 Vlachopoulos C, Aznaouridis K, Stefanadis C. Prediction of cardiovascular events and all-cause mortality with arterial stiffness. J Am Coll Cardiol 2010; 55: 1318-1327.

12 Cruickshank K, Riste L, Anderson S, Wright J, Dunn G, Gosling R. Aortic pulse wave velocity and its relationship to mortality in diabetes and glucose intolerance. Circulation 2002; 106: 2085-2090.

13 Vyssoulis GP, Pietri PG, Karpanou EA, Vlachopoulos CV, Kyvelou SM, Spanos P, Cokkinos DV, Stefanadis Cl. Differential impact of metabolic syndrome on arterial stiffness and wave reflections: focus on distinct definitions. Int J Cardiol 2010; 138 119-125.

14 Schillaci G, Pirro M, Vaudo G, Mannarino M, Savarese G, Pucci G, Franklin S, Mannarino E. Metabolic syndrome is associated with aortic stiffness in untreated essential hypertension. Hypertension 2005; 45: 1078-1082.

15 Czernichow S, Bertrais S, Blacher J, Oppert J-M, Galan P, Ducimetièr P, Hercberg S, Safar S, Zureik M. Metabolic syndrome in relation to structure and function of large arteries: a predominant effect of blood pressure. A report from the SU.VI.MAX Vascular Study. Am J Hypertens 2005; 18: 1154-1160.

16 Mulè G, Cottone S, Mongiovì R, Cusimano P, Mezzatesta G, Seddio G, Volpe V, Nardi E, Andronico G, Piazza G, Cerasola G. Influence of the metabolic syndrome on aortic stiffness in never treated hypertensive patients. Nutr Metab Cardiovasc Dis 2006; 16: 54-59.

17 Scuteri A, Najjar S, Muller D, Andres R, Hougaku H, Metter J, Lakatta E. Metabolic syndrome amplifies the age-associated increases in vascular thickness and stiffness. J Am Coll Cardiol 2004; 43: 1388-1395.

18 Ferreira I, Boreham C, Twisk J, Gallagher A, Young I, Murray L, Stehouwer C. Clustering of metabolic syndrome risk factors and arterial stiffness in young adults: the Northern Ireland Young Heart Project. J Hypertens 2007; 25: 1009-1020.

19 Protogerou A, Blacher J, Aslangul E, Le Jeunne C, Lekakis J, Mavrikakis M, Safar M. Gender influence on metabolic syndrome's effects on arterial stiffness and pressure wave reflections in treated hypertensive subjects. Atherosclerosis 2007; 193 . 151-158.

20 Pedrinelli R, Giampietro O, Carmassi F, Melillo E, Dell'Omo G, Catapano G, Matteucci E, Talarico L, Morale M, De Negri F, Di Bello V. Microalbuminuria and endothelial dysfunction in essential hypertension. Lancet 1994; 344: 14-18.

21 Gerstein H, Mann J, Yi Q, Dinneen S, Hoogwerf B, Hallé JP, Young J, Rashkow A, Joyce C, Nawaz S, Yusuf Sfor the HOPE Study Investigators. Albuminuria and risk of cardiovascular events, death and heart failure in diabetic and nondiabetic individuals. JAMA 2001; 286: 421-426.

22 Alberti KG, Zimmet PZ. Definition, diagnosis and classification of diabetes mellitus and its complications. Part 1: diagnosis and classification of diabetes mellitus provisional report of a WHO consultation. Diabet Med 1998; 15: 539-553.
23 Laurent S, Cockcroft J, Van Bortel L, Boutouyrie P, Giannatasio C, Hayoz D, Pannier B, Vlachopoulos C, Wilkinson I, Struijker-Boudier H. On behalf of the European Network for Non-invasive investigation of Large Arteries. Expert consensus document on arterial stiffness: methological issues and clinical applications. Eur Heart J 2006; 27: 2588-2605.

24 Asmar R, Benetos A, Topouchian J, Laurent S, Pannier B, Brisac A-M, Target R, Levy BI. Assessment of arterial distensibility by automatic pulse wave velocity measurement: validation and clinical application studies. Hypertension 1995; 26: 485-490.

25 Scuteri A, Najiar S, Orru M, Usala G, Piras MG, Ferrucci L, Cao A, Schlessinger D, Uda M, Lakatta E. The central arterial burden of the metabolic syndrome is similar in men and women: the SardiNIA Study. Eur Heart J 2010; 31: 602-613.

26 Tomiyama H, Koji Y, Yambe M, Motobe K, Shiina K, Gulnisa Z, Yamamoto Y, Yamashina A. Elevated C-reactive protein augments increased arterial stiffness in subjects with the metabolic syndrome. Hypertension 2005; 45: 997-1003.

27 Levy D, Hwang S-J, Kayalar A, Benjamin E, Vasan R, Parise H, Larson M, Wang T, Selhub J, Jacques P, Vita J, Keyes M, Mitchell G. Associations of plasma natriuretic peptide, adrenomedullin, and homocysteine levels with alterations in arterial stiffness: The Framingham Heart Study. Circulation 2007; 115: 3079-3085.

28 Bortolotto L, Safar M, Billaud E, Lacroix C, Asmar R, London G, Blacher J. Plasma homocysteine, aortic stiffness, and renal function in hypertensive patients. Hypertension 1999; 34: 837-842.

29 Tsioufis C, Dimitriadis K, Andrikou E, Thomopoulos C, Tsiachris D, Stefanadi E, Mihas C, Miliou A, Papademetriou V, Stefanadis C. ADMA, C-reactive protein and albuminuria in untreated essential hypertension: a cross-sectional study. Am J Kidney Dis 2010; 55: 1050-1059.

30 Jager A, Kostense P, Nijpels G, Dekker J, Heine R, Bouter L, Donker A, Stehouwer C. Serum homocysteine levels are associated with the development of (micro)albuminuria. The Hoorn Study. Arterioscler Thromb Vasc Biol 2001; 21: 74-81.

31 Pravenec M, Kajiya T, Zídek V, Landa V, Mlejnek P, Simáková M, Silhavý J, Malínská H, Oliyarnyk O, Kazdová L, Fan J, Wang J, Kurtz T. Effects of human C-reactive protein on pathogenesis of features of the metabolic syndrome. Hypertension 2011; 57: 731-737.

32 Zhao Z, Nie H, He H, Yan Z, Liu D, Luo Z, Ma L, Ni Y, Chen J, Jing J, Cao T, Yang $\mathrm{H}$, Tepel M, Zhang W, Zhu Z. High-sensitivity C-reactive protein predicts target organ damage in Chinese patients with metabolic syndrome. Metabolism 2007; 56: 1612-1619.

33 Pedrinelli R, Dell'Omo G, Di Bello V, Pellegrini G, Pucci L, Del Prato S, Penno G. Lowgrade inflammation and microalbuminuria in hypertension. Atheroscler Thromb Vasc Biol 2004; 24: 2414-2419.

34 Hashimoto J, Ito S. Central pulse pressure and aortic stiffness determine renal hemodynamics: pathophysiological implication for microalbuminuria in hypertension. Hypertension 2011; 58: 839-846.

35 O'Rourke M, Safar M. Relationship between aortic stiffening and microvascular disease in brain and kidney: cause and logic of therapy. Hypertension 2005; 46: 200-204.

36 Wang Y, Hu Y, Li Y, Chu S, Zhu D, Gao P. Association of renal function with the ambulatory arterial stiffness index and pulse pressure in hypertensive patients. Hypertens Res 2012; 35: 201-206.

37 Munakata M, Miura Y, Yoshinaga K. On behalf of the J-TOPP study group. Highe brachial-ankle pulse wave velocity as an independent risk factor for future microalbuminuria in patients with essential hypertension: the J-TOPP study. J Hypertens 2009; 27: 1466-1471.

38 Vlachopoulos C, Dima I, Aznaouridis K, Vasiliadou C, loakeimidis N, Aggeli C, Toutouza M, Stefanadis $C$. Acute systemic inflammation increases arterial stiffness and decreases wave reflections in healthy individuals. Circulation 2005; 112: 2193-2200.

39 Hingorani AD, Cross J, Kharbanda RK, Mullen M, Bhagat K, Taylor M, Donald AE, Palacios M, Griffin GE, Deanfield JE, Chir B, MacAllister RJ, Vallance P. Acute systemic inflammation impairs endothelium-dependent dilatation in humans. Circulation 2000; 102: 994-999. 